\title{
An Operator-Based Approach for the Construction of Closed-Form Solutions to Fractional Differential Equations
}

\section{Zenonas Navickas ${ }^{a}$, Tadas Telksnys ${ }^{a}$, Inga Timofejeva ${ }^{a}$, Romas Marcinkevičius ${ }^{b}$ and Minvydas Ragulskis ${ }^{a}$}

\author{
${ }^{a}$ Research Group for Mathematical and Numerical Analysis of Dynamical \\ Systems, Kaunas University of Technology \\ Studentu 50-147, Kaunas LT-51368, Lithuania \\ ${ }^{b}$ Department of Software Engineering, Kaunas University of Technology \\ Studentu 50-415, Kaunas LT-51368, Lithuania \\ E-mail(corresp.): tadas.telksnys@ktu.1t
}

Received March 1, 2018; revised September 13, 2018; accepted September 13, 2018

\begin{abstract}
An operator-based approach for the construction of closed-form solutions to fractional differential equations is presented in this paper. The technique is based on the analysis of Caputo and Riemann-Liouville algebras of fractional power series. Explicit solutions to a class of linear fractional differential equations are obtained in terms of Mittag-Leffler and fractionally-integrated exponential functions in order to demonstrate the viability of the proposed technique.
\end{abstract}

Keywords: fractional differential equation, operator calculus, analytical solution, closedform solution.

AMS Subject Classification: 34A08; 34A25; $26 \mathrm{~A} 33$.

\section{Introduction}

Fractional differential equations (FDEs) have become one of the cornerstones in the modeling of various real-world systems in recent years. Fractional-order models are extensively utilized in the study of physics [17], nanomaterials [9], control problems in engineering [24], economics [14] and biomedicine [21].

Several typical recent examples of research involving fractional calculus are reviewed below. A fractional calculus model of supercapacitor energy storage is presented in [18]. Viscoelastic constitutive laws for arterial wall mechanics are investigated via fractional-order models in [36]. Atomic chain dynamics 
are approximated using fractional differential equations in [32]. Dynamics of the transition from laminar to turbulent fluid flow is described using fractional models in [15].

A fractional-order model of anomalous cosmic ray diffusion with a finite velocity of free particle motion is considered in [34]. An efficient fractionalderivative based model for the prediction of multiaxial visco-hyperelastic behavior of elastomers is constructed in [8]. Fractional dynamical systems with types of attractors that are distinct from attractors of integer-order systems are considered in [10]. It is demonstrated in [20] that the dependence of the firing rate of single rat neocortical pyramidal neuron is a fractional derivative of slowly varying stimulus parameters.

Due to such wide possibilities fractional-order model application, a number of methods for the construction of exact analytical solutions to FDEs have been developed. In [35], the Carleman embedding technique is used to transform the fractional logistic equation into an infinite-order set of linear equations from which the exact solution to the fractional logistic equation is obtained. Agrawal presents a scheme for the construction of analytical solutions for a class of FDEs that contain both left- and right-fractional derivatives in [1]. The joint Laplace and Fourier transform is employed to construct solutions to fractional partial differential equations occurring in quantum mechanics in [29]. A class of explicit particular solutions to the Cauchy-Euler fractional partial differential equation is obtained in [19].

Analytical solutions to the fractional modified Telegraph and Rayleigh equations are constructed in terms of Mittag-Leffler, Hypergeometric, Hermite and Fox's $\mathrm{H}$ functions in [33]. The solution to a Hilfer-generalized Riemann-Liouville fractional diffusion equation is obtained using variable separation, Laplace transform and Sturm-Liouville analysis in [31]. A bivariate operational method is used to construct solution to the two-term time-fractional Thornley problem in [7]. Exact solutions to a class of fractional Hamiltonian equations are analyzed in [6]. An extension of Frobenius' method is applied to linear fractional differential equations with variable coefficients in [30].

A number of techniques use power series with fractional powers to construct solutions to fractional differential equations. The viability of this approach is proven in [11] by generalizing some results from integer-power series to fractional-power series using the Caputo fractional derivative. A new approach for the iterated construction of series solutions to fractional differential equations is presented in [2] and expanded in [3].

An analytical technique based on power series is exploited in [5] to predict and represent the multiplicity of solutions to nonlinear boundary value problems of fractional order. This approach is further generalized in [12] and [4] in which the authors propose schemes for the construction of exact analytical solutions to linear and nonlinear equations based on the generalized Taylor series formula.

The main objective of this paper is to develop an operator-based approach for the construction of closed-form solutions to fractional differential equations. Some key concepts of such operator-based techniques are presented in [26] for fractional derivative order $\alpha=\frac{1}{2}$. A generalization of this technique for 
rational-valued fractional derivative order $\alpha=\frac{m}{n}$ is presented in this paper.

The presented technique is based on Caputo and Riemann-Liouville algebras of fractional power series. Fractional differentiation and integration operators are defined using the basis of these algebras. It is demonstrated that a one-toone correspondence exists between Caputo and Riemann-Liouville series.

To provide a more concise and clear presentation of these concepts, linear fractional differential equations are considered. Using the properties of Caputo and Riemann-Liouville algebras, explicit expressions of solutions to linear fractional differential equations are obtained via linear recurring sequences. Furthermore, it is shown that elements of Caputo and Riemann-Liouville algebras can be represented by a finite sum of fractionally integrated integer-power series, which enables the transformation of fractional differential equations into systems of ordinary differential equations (ODEs). The viability of our approach is demonstrated using the fractional damped harmonic oscillator - it is shown that as the fractional derivative order approaches the standard integer value, the exact solution of the FDE converges to the exponential-function exact solution of the respective ODE.

\section{Main concepts and definitions}

A generalization of algebras and operators given in [26] for fractional derivatives of order $\alpha=\frac{1}{n}, n \in \mathbb{N}$ is presented in this section.

\subsection{Power series and extensions}

As in [26], functions in this paper are represented by power series:

$$
y(z):=\sum_{j=0}^{+\infty} a_{j} \frac{z^{j}}{j !} ; \quad a_{j}, z \in \mathbb{C} .
$$

If series (2.1) is convergent (in the Cauchy sense) in any ball $|z|<\varepsilon$, classical function extension techniques can be used to extend (2.1) to a wider domain in the complex plane. Denoting $y(x), x \in \mathbb{R}$ as a real-valued function that is obtained by evaluating the extended power series on the real line allows to consider (2.1) for arguments that are not necessarily in the convergence radius $|x|<\varepsilon$. In such cases, the extended function $y(x)$ and the respective power series are considered congruent.

If series (2.1) is divergent for $z \neq 0$, it is well-known that while such series cannot be evaluated, they still contain important structural information [16]. Thus, divergent series can be solutions to fractional differential equations in the structural sense.

\subsection{Fractional power series}

Solutions to fractional differential equations can be expressed via power series that are summed over non-integer powers. In the remainder of this paper, we let $x \geq 0$. 
Definition 1 . Let $n \in \mathbb{N}$. The fractional power series basis $z_{0}^{(n)}, z_{1}^{(n)}, \ldots$ is defined as:

$$
z_{j}^{(n)}(x):=x^{\frac{j-n+1}{n}} / \Gamma\left(\frac{j+1}{n}\right) ; \quad x \geq 0 ; \quad j=0,1, \ldots
$$

$\Gamma$ denotes the Gamma function:

$$
\Gamma(x):=\int_{0}^{+\infty} \xi^{x-1} \exp (-\xi) \mathrm{d} \xi
$$

DEFINITION 2. The set of fractional power series with respect to parameter $n \in \mathbb{N}$ reads:

$$
\mathbb{F}_{n}:=\left\{\sum_{j=0}^{+\infty} c_{j} z_{j}^{(n)} ; \quad c_{j} \in \mathbb{C}, j=0,1, \ldots\right\}
$$

\subsection{Riemann-Liouville algebra}

Consider the linear space over $\mathbb{C}$ defined in the set $\mathbb{F}_{n}$ with standard sum and product by a scalar operations. Let $z_{k}^{(n)}, z_{l}^{(n)} ; k, l=0,1, \ldots$ be basis elements of any fractional power series.

Definition 3. The Riemann-Liouville type product operation $*_{n}$ in the set $\mathbb{F}_{n}$ is defined as:

$$
z_{k}^{(n)} *_{n} z_{l}^{(n)}:=\left(\begin{array}{c}
(k+l) / n \\
k / n
\end{array}\right) z_{k+l}^{(n)},
$$

where $\left(\begin{array}{l}\alpha \\ \beta\end{array}\right)$ is the generalized binomial coefficient:

$$
\left(\begin{array}{l}
\alpha \\
\beta
\end{array}\right)=\frac{\Gamma(\alpha+1)}{\Gamma(\beta+1) \Gamma(\alpha+\beta-1)} ; \quad \alpha, \beta \in \mathbb{C} .
$$

Note that (2.3) is not the natural product that is obtained by multiplying basis functions in a conventional way, since that would yield powers of $x$ that are lesser than negative unity.

Using relation $(2.3)$, the product of any $f_{1}^{(n)}=\sum_{j=0}^{+\infty} a_{j} z_{j}^{(n)}$, $f_{2}^{(n)}=\sum_{j=0}^{+\infty} b_{j} z_{j}^{(n)} \in \mathbb{F}_{n}$ is defined as:

$$
\begin{aligned}
f_{1}^{(n)} *_{n} f_{2}^{(n)} & :=\sum_{j=0}^{+\infty} \sum_{k=0}^{j} a_{k} b_{j-k} z_{k}^{(n)} *_{n} z_{j-k}^{(n)} \\
& =\sum_{j=0}^{+\infty}\left(\sum_{k=0}^{j} a_{k} b_{j-k}\left(\begin{array}{l}
j / n \\
k / n
\end{array}\right)\right) z_{j}^{(n)} \in \mathbb{F}_{n} .
\end{aligned}
$$

The neutral element with respect to $*_{n}$ is $z_{0}^{(n)}$. Furthermore, $*_{n}$ is distributive with respect to the conventional sum operation, thus the linear space $\mathbb{F}_{n}$ together with product $*_{n}$ defines an algebra. 
Definition 4. The commutative algebra $\mathcal{F}_{n}:=\left\langle\mathbb{F}_{n} ;+, *_{n} \mid \mathbb{C}\right\rangle$ is called the Riemann-Liouville algebra.

Definition 5. Riemann-Liouville fractional differentiation and integration operators are defined for elements of $\mathcal{F}_{n}$ in the classical sense [22,27]:

$$
\mathbf{D}^{(1 / n)} z_{j}^{(n)}:=\left\{\begin{array}{ll}
0, & j=0 ; \\
z_{j-1}^{(n)}, & j=1,2, \ldots,
\end{array} \quad \mathbf{I}^{(1 / n)} z_{j}^{(n)}:=z_{j+1}^{(n)}, j=0,1, \ldots\right.
$$

Note that Riemann-Liouville differentiation of a constant does not result in zero, since $\mathbf{D}^{(1 / n)} 1=\mathbf{D}^{(1 / n)} z_{n-1}^{(n)}=z_{n-2}^{(n)}$. It is clear that $\mathbf{D}^{(1 / n)} f, \mathbf{I}^{(1 / n)} f \in \mathbb{F}_{n}$ for any $f \in \mathbb{F}_{n}$.

\subsection{Caputo algebra}

Consider the truncated set of basis functions (2.2), starting at $z_{n-1}^{(n)}=1$ :

$$
w_{j}^{(n)}:=z_{j+n-1}^{(n)}, \quad j=0,1, \ldots
$$

Definition 6. Fractional power series constructed using the basis $w_{0}^{(n)}, w_{1}^{(n)}$, $w_{2}^{(n)}, \ldots$ comprise the set of Caputo power series:

$$
{ }^{C} \mathbb{F}_{n}:=\left\{\sum_{j=0}^{+\infty} c_{j} w_{j}^{(n)} ; \quad c_{j} \in \mathbb{C}, \quad j=0,1, \ldots\right\} .
$$

As in the previous subsection, the set of Caputo power series forms a linear space over $\mathbb{C}$ with conventional sum and product by a scalar operations. Since powers of $x$ in the set ${ }^{C} \mathbb{F}_{n}$ are non-negative, the standard algebraic product operation can be used to define products of basis functions:

$$
w_{k}^{(n)} w_{l}^{(n)}=\left(\begin{array}{c}
\frac{k+l}{n} \\
\frac{k}{n}
\end{array}\right) w_{k+l}^{(n)} ; \quad k, l=0,1, \ldots
$$

By $(2.5)$, the product of any $g_{1}^{(n)}=\sum_{j=0}^{+\infty} a_{j} w_{j}^{(n)}, g_{2}^{(n)}=\sum_{j=0}^{+\infty} b_{j} w_{j}^{(n)} \in{ }^{C} \mathbb{F}_{n}$ is defined as:

$$
g_{1}^{(n)} g_{2}^{(n)}=\sum_{j=0}^{+\infty}\left(\sum_{k=0}^{j} a_{k} b_{j-k}\left(\begin{array}{l}
j / n \\
k / n
\end{array}\right)\right) w_{j}^{(n)} \in{ }^{C} \mathbb{F}_{n}
$$

The neutral element with respect to $(2.6)$ is $w_{0}^{(n)}=1$. It is clear that standard algebraic sum, product and product by a scalar operations define an algebra in the set ${ }^{C} \mathbb{F}_{n}$.

Definition 7 . The algebra ${ }^{C} \mathcal{F}_{n}:=\left\langle{ }^{C} \mathbb{F}_{n} ;+, \cdot \mid \mathbb{C}\right\rangle$ is called the Caputo algebra. 
DEFinition 8. Caputo differentiation and integration operators are defined for any $g \in{ }^{C} \mathbb{F}_{n}$ via the following relations:

$$
{ }^{C} \mathbf{D}^{(1 / n)} w_{j}^{(n)}=\left\{\begin{array}{ll}
0, & j=0, \\
w_{j-1}^{(n)}, & j=1,2, \ldots,
\end{array} \quad{ }^{C} \mathbf{I}^{(1 / n)} w_{j}^{(n)}=w_{j+1}^{(n)}, \quad j=0,1, \ldots\right.
$$

Note that the Caputo differentiation of unity is equal to zero, since ${ }^{C} \mathbf{D}^{(1 / n)} w_{0}^{(n)}=0$. The set ${ }^{C} \mathbb{F}_{n}$ is closed with respect to operators ${ }^{C} \mathbf{D}^{(1 / n)}$, ${ }^{C} \mathbf{I}^{(1 / n)}$.

\subsection{Relationship between Riemann-Liouville and Caputo algebras and operators}

\subsubsection{Relationship between algebras with equal differentiation order}

It has already been noted that the set ${ }^{C} \mathbb{F}_{n}$ consists of a subset of basis functions from the set $\mathbb{F}_{n}$. Let $\tau$ define the following mapping:

$$
\tau\left(z_{j}^{(n)}\right)=w_{j}^{(n)} ; \quad \tau^{-1}\left(w_{j}^{(n)}\right)=z_{j}^{(n)} ; \quad j=0,1, \ldots
$$

Then, $\tau$ is a bijection between sets $\mathbb{F}_{n}$ and ${ }^{C} \mathbb{F}_{n}$. Note that:

$$
\begin{aligned}
\tau\left(z_{k}^{(n)} *_{n} z_{l}^{(n)}\right) & =\tau\left(z_{k}^{(n)}\right) \tau\left(z_{l}^{(n)}\right)=w_{k}^{(n)} w_{l}^{(n)}, \\
\tau^{-1}\left(w_{k}^{(n)} w_{l}^{(n)}\right) & =\tau^{-1}\left(w_{k}^{(n)}\right) *_{n} \tau^{-1}\left(w_{l}^{(n)}\right)=z_{k}^{(n)} *_{n} z_{l}^{(n)},
\end{aligned}
$$

for $k, l=0,1, \ldots$ Equations (2.8), (2.9) yield that:

$$
\tau\left(f_{1} *_{n} f_{2}\right)=\tau\left(f_{1}\right) \tau\left(f_{2}\right) ; \quad \tau^{-1}\left(g_{1} g_{2}\right)=\tau^{-1}\left(g_{1}\right) *_{n} \tau^{-1}\left(g_{2}\right),
$$

for any $f_{1}, f_{2} \in \mathbb{F}_{n}, g_{1}, g_{2} \in C^{C} \mathbb{F}_{n}$. Thus the mapping (2.7) defines a bijection between algebras $\mathcal{F}_{n}$ and ${ }^{C} \mathcal{F}_{n}$.

It can be observed that the mappings $\tau, \tau^{-1}$ can be realized via operators $\mathbf{D}^{(1 / n)}, \mathbf{I}^{(1 / n)}$ :

$$
\left.\tau\right|_{\mathbb{F}_{n}}=\left(\mathbf{I}^{(1 / n)}\right)^{n-1},\left.\quad \tau^{-1}\right|_{C_{\mathbb{F}_{n}}}=\left(\mathbf{D}^{(1 / n)}\right)^{n-1}
$$

The relationship between algebras $\mathcal{F}_{n}$ and ${ }^{C} \mathcal{F}_{n}$ is summarized in Figure 1. Note that:

$$
\left.{ }^{C} \mathbf{D}^{(1 / n)}\right|_{C_{\mathbb{F}_{n}}}=\left.\left(\mathbf{I}^{(1 / n)}\right)^{n-1}\left(\mathbf{D}^{(1 / n)}\right)^{n}\right|_{C_{\mathbb{F}_{n}}},\left.\quad{ }^{C} \mathbf{I}^{(1 / n)}\right|^{C} \mathbb{F}_{n}=\left.\mathbf{I}^{(1 / n)}\right|_{C_{\mathbb{F}_{n}}} .
$$

Furthermore, if $f_{k} \in \mathbb{F}_{n}$ and $g_{k} \in{ }^{C} \mathbb{F}_{n}, k=1, \ldots, m$, then:

$$
\left(\mathbf{I}^{(1 / n)}\right)^{n-1}\left(f_{1} *_{n} f_{2} *_{n} \ldots *_{n} f_{m}\right)=\prod_{k=1}^{m}\left(\left(\mathbf{I}^{(1 / n)} f_{k}\right)^{n-1}\right)
$$




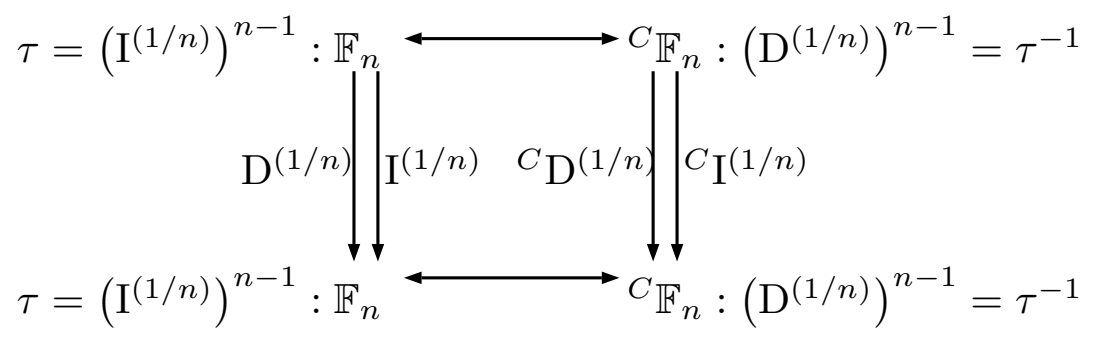

Figure 1. Schematic diagram of the bijective mappings between Caputo and Riemann-Liouville algebras.

$$
\begin{aligned}
\left(\mathbf{D}^{(1 / n)}\right)^{n-1} & \left(\prod_{k=1}^{m} g_{k}\right)=\left(\left(\mathbf{D}^{(1 / n)}\right)^{n-1} g_{1}\right) *_{n}\left(\left(\mathbf{D}^{(1 / n)}\right)^{n-1} g_{2}\right) *_{n} \ldots \\
& *_{n}\left(\left(\mathbf{D}^{(1 / n)}\right)^{n-1} g_{m}\right) .
\end{aligned}
$$

Note that the mapping $\tau$ can be used to exchange Caputo differentiation with Riemann-Liouville and vice-versa:

$$
\tau\left(\left(\mathbf{D}^{(1 / n)}\right)^{m} g\right)=\left({ }^{C} \mathbf{D}^{(1 / n)}\right)^{m} f, \quad \tau^{-1}\left(\left({ }^{C} \mathbf{D}^{(1 / n)}\right)^{m} f\right)=\left(\mathbf{D}^{(1 / n)}\right)^{m} g,
$$

where $f \in C^{C} \mathbb{F}_{n} ; g=\tau^{-1}(f) \in \mathbb{F}_{n}$ and $m=0,1, \ldots$

\subsubsection{Relationship between algebras with distinct differentiation order}

Let us consider the fractional derivative order parameter $n$ factored into powers of primes $p_{1}, \ldots, p_{m}$ :

$$
n=\prod_{j=1}^{m} p_{j}^{k_{j}}, \quad m, k_{j} \in \mathbb{N} .
$$

The following relations hold true for Caputo fractional power series:

$$
{ }^{C} \mathbb{F}_{p_{j}} \subset \cdots \subset{ }^{C} \mathbb{F}_{p_{j} k_{j}} \subseteq{ }^{C} \mathbb{F}_{n},
$$

however, in general, ${ }^{C} \mathbb{F}_{p_{1}} \cup{ }^{C} \mathbb{F}_{p_{1}^{2}} \cup \cdots \cup \cup^{C} \mathbb{F}_{p_{m}^{k_{m}}} \neq{ }^{C} \mathbb{F}_{n}$. Furthermore, ${ }^{C} \mathbb{F}_{p_{j}} \cap$ ${ }^{C} \mathbb{F}_{p_{l}}={ }^{C} \mathbb{F}_{1}, j \neq l$. The set ${ }^{C} \mathbb{F}_{1}$ contains basis elements with integer powers, thus, the unit element $w_{0}^{\left(p_{j}\right)}=w_{0}^{\left(p_{l}\right)}=w_{0}^{(n)}=1$ is the same for any subset ${ }^{C} \mathbb{F}_{p_{j}^{l}}, l=1, \ldots, k_{j}$. This leads to the fact that algebras formed from the sets given in (2.10) are subalgebras of ${ }^{C} \mathcal{F}_{n}$ :

$$
{ }^{C} \mathcal{F}_{p_{j}} \subset \cdots \subset{ }^{C} \mathcal{F}_{p_{j}} \subseteq{ }^{C} \mathcal{F}_{n} .
$$

In the case of Riemann-Liouville derivative, the relation analogous to (2.10) holds true:

$$
\mathbb{F}_{p_{j}} \subset \cdots \subset \mathbb{F}_{p_{j}^{k_{j}}} \subseteq \mathbb{F}_{n}
$$


However, in this case the unit elements are distinct for each algebra formed with the subsets $\mathbb{F}_{p_{j}^{l}}, l=1, \ldots, k_{j}$. Furthermore, the definition of the product (2.4) depends on the order of the algebra. These observations yield that $\mathcal{F}_{p_{j}^{l}}$ are not sub-algebras of $\mathcal{F}_{n}$.

An example of the results of this subsection for $n=6$ is given in Figure 2. Note that ${ }^{C} \mathcal{F}_{2},{ }^{C} \mathcal{F}_{3}$ are subalgebras of ${ }^{C} \mathcal{F}_{6}$, but $\mathcal{F}_{2}, \mathcal{F}_{3}$ are not subalgebras of $\mathcal{F}_{6}$, even though $\mathbb{F}_{2}, \mathbb{F}_{3} \subset \mathbb{F}_{6}$. The bijection $\tau$ maps Caputo and RiemannLiouville algebras of the same order to each other.

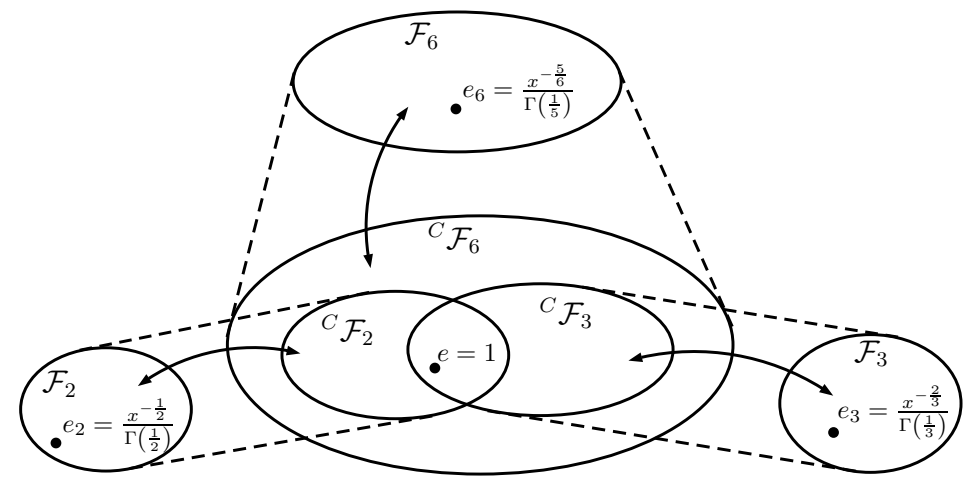

Figure 2. An example of the relationship between Caputo and Riemann-Liouville algebras of orders $n=2,3,6$. Caputo algebras ${ }^{C} \mathcal{F}_{2},{ }^{C} \mathcal{F}_{3}$ are subalgebras of ${ }^{C} \mathcal{F}$. Riemann-Liouville fractional power series sets satisfy $\mathbb{F}_{2}, \mathbb{F}_{3} \subset \mathbb{F}_{6}$, but Riemann-Liouville algebras $\mathcal{F}_{2}, \mathcal{F}_{3}$ are not subalgebras of $\mathcal{F}_{6}$ due to the fact that the product operation $*_{n}$ depends on algebra order. $e$ denotes the unit element of all considered Caputo algebras; $e_{2}, e_{3}, e_{6}$ denote unit elements of Riemann-Liouville algebras that correspond to respective fractional differentiation orders.

\section{Linear fractional differential equations}

Linear fractional differential equations with respect to Caputo differentiation operators are analyzed in this section.

\subsection{Mittag-Leffler functions}

Mittag-Leffler functions, first introduced in [23], play a pivotal role in fractional calculus.

Definition 9. Let $\alpha, \beta \in \mathbb{C}$ and $\operatorname{Re}(\alpha)>0$. Then, the Mittag-Leffler function is defined as [28]:

$$
E_{\alpha, \beta}(t):=\sum_{j=0}^{+\infty} \frac{t^{j}}{\Gamma(\alpha j+\beta)} .
$$

Special cases of (3.1) include exponent and hyperbolic functions. 
Note that setting $\alpha=1 / n, \beta=1, t=x^{\frac{1}{n}}$, where $n \in \mathbb{N}$ results in:

$$
E_{\frac{1}{n}, 1}\left(x^{\frac{1}{n}}\right)=\sum_{j=0}^{+\infty} w_{j}^{(n)} .
$$

Thus, the Mittag-Leffler functions can be considered as the analogy of exponential functions for fractional differential operators, since they are the sum of all basis elements. Furthermore, the following relation holds true for any $\rho \in \mathbb{C}:$

$$
E_{\frac{1}{n}, 1}\left(\rho x^{\frac{1}{n}}\right)=\sum_{j=0}^{+\infty} \rho^{j} w_{j}^{(n)},
$$

which leads to:

$$
{ }^{C} \mathbf{D}^{(1 / n)} E_{\frac{1}{n}, 1}\left(\rho x^{\frac{1}{n}}\right)=\rho E_{\frac{1}{n}, 1}\left(\rho x^{\frac{1}{n}}\right) .
$$

Let $s=0,1, \ldots$. The following relation will be used to express solutions to linear differential equations in the Caputo algebra:

$$
\begin{gathered}
\frac{\mathrm{d}^{s}}{\mathrm{~d} \rho^{s}} E_{\frac{1}{n}, 1}\left(\rho x^{\frac{1}{n}}\right)=\sum_{j=0}^{+\infty}\left(\frac{\mathrm{d}^{s}}{\mathrm{~d} \rho^{s}} \rho^{j}\right) w_{j}^{(n)}=\sum_{j=s}^{+\infty} \frac{j !}{(j-s) !} \rho^{j-s} w_{j}^{(n)} \\
=s ! \sum_{j=0}^{+\infty}\left(\begin{array}{l}
j \\
s
\end{array}\right) \rho^{j-s} w_{j}^{(n)} .
\end{gathered}
$$

Note that $\left(\begin{array}{l}j \\ s\end{array}\right)=0$ when $j<s$ and $j, s \in \mathbb{N} \cup\{0\}$. Equation (3.4) yields:

$$
\sum_{j=0}^{+\infty}\left(\begin{array}{l}
j \\
s
\end{array}\right) \rho^{j-s} w_{j}^{(n)}=\frac{1}{s !} \frac{\mathrm{d}^{s}}{\mathrm{~d} \rho^{s}} E_{\frac{1}{n}, 1}\left(\rho x^{\frac{1}{n}}\right) .
$$

\subsection{Linear equations with constant coefficients}

Consider the following differential equation with respect to $y \in{ }^{C} \mathbb{F}$ :

$$
\left({ }^{C} \mathbf{D}^{(1 / n)}\right)^{m} y+b_{m-1}\left({ }^{C} \mathbf{D}^{(1 / n)}\right)^{m-1} y+\cdots+b_{1}{ }^{C} \mathbf{D}^{(1 / n)} y+b_{0} y=f
$$

where $f=\sum_{j=0}^{+\infty} f_{j} w_{j}^{(n)} \in C_{\mathbb{F}}$ is a known function; $b_{k} \in \mathbb{C}, k=0, \ldots, m-1$ and $n, m \in \mathbb{N}$.

Note that equation (3.6) can be transformed into a Riemann-Liouville differential equation using the previously described mapping $\tau^{-1}$. Letting $\widehat{y}=$ $\tau^{-1}(y)$ and applying the mapping $\tau^{-1}$ on both sides of (3.6) yields:

$$
\left(\mathbf{D}^{(1 / n)}\right)^{m} \widehat{y}+b_{m-1}\left(\mathbf{D}^{(1 / n)}\right)^{m-1} \widehat{y}+\cdots+b_{1} \mathbf{D}^{(1 / n)} \widehat{y}+b_{0} \widehat{y}=\tau^{-1}(f) .
$$

Similarly, applying $\tau$ to both sides of (3.7) results in (3.6). Thus it is sufficient to consider Caputo differential equations (3.6). 


\subsubsection{Homogeneous case}

Consider the case $f=0$. Let $y=\sum_{j=0}^{+\infty} c_{j} w_{j}^{(n)}$, where $c_{j} \in \mathbb{C}$ are undetermined coefficients. Note that:

$$
\left({ }^{C} \mathbf{D}^{(1 / n)}\right)^{k} y=\sum_{j=0}^{+\infty} c_{j+k} w_{j}^{(n)}, \quad k=0,1, \ldots
$$

Inserting the expression of $y$ into (3.6) and using (3.8) yields:

$$
\sum_{j=0}^{+\infty}\left(c_{j+m}+b_{m-1} c_{j+m-1}+\cdots+b_{1} c_{j+1}+b_{0} c_{j}\right) w_{j}^{(n)}=0 .
$$

Thus, the coefficients of the solution must satisfy:

$$
c_{j+m}+b_{m-1} c_{j+m-1}+\cdots+b_{1} c_{j+1}+b_{0} c_{j}=0, \quad j=0,1, \ldots
$$

Equation (3.9) defines a linear recurrence relation, which can be solved by considering roots of the following characteristic polynomial:

$$
P(\rho)=\rho^{m}+b_{m-1} \rho^{m-1}+\cdots+b_{1} \rho+b_{0}=0 .
$$

Suppose (3.10) has roots $\rho_{1}, \ldots, \rho_{l}$ with multiplicities $l_{1}, \ldots, l_{l}$. Then, the solution to (3.9) takes the following form $[13,25]$ :

$$
c_{j}=\sum_{k=1}^{l} \sum_{s=0}^{l_{k}} \gamma_{k s}\left(\begin{array}{l}
j \\
s
\end{array}\right) \rho_{k}^{j-s}, \quad j=0,1, \ldots,
$$

where $\gamma_{k s} \in \mathbb{C}$ are constants that can be determined from a system of linear equations which is obtained by selecting $j=0, \ldots, l-1$ in (3.11). Note that $0^{0}:=1$ and the product $\left(\begin{array}{l}j \\ s\end{array}\right) \rho_{k}^{j-s}$ is considered to be zero if at least one of its terms is equal to zero.

Inserting (3.11) into the expression of $y$ and using (3.5) yields:

$$
\begin{aligned}
y & =\sum_{j=0}^{+\infty}\left(\sum_{k=1}^{l} \sum_{s=0}^{l_{k}} \gamma_{k s}\left(\begin{array}{l}
j \\
s
\end{array}\right) \rho_{k}^{j-s}\right) w_{j}^{(n)}=\sum_{k=1}^{l} \sum_{s=0}^{l_{k}} \gamma_{k s}\left(\sum_{j=s}^{+\infty}\left(\begin{array}{l}
j \\
s
\end{array}\right) \rho_{k}^{j-s} w_{j}^{(n)}\right) \\
& =\left.\sum_{k=1}^{l} \sum_{s=0}^{l_{k}} C_{k s}\left(\frac{\mathrm{d}^{s}}{\mathrm{~d} \rho^{s}} E_{\frac{1}{n}, 1}\left(\rho x^{\frac{1}{n}}\right)\right)\right|_{\rho=\rho_{k}},
\end{aligned}
$$

where $C_{k s}=\frac{1}{s !} \gamma_{k s}$. The results of this subsection can be summarized with the following Lemma.

Lemma 1. Let a linear homogeneous fractional differential equation with constant coefficients with respect to the Caputo derivative be given:

$$
\left({ }^{C} \mathbf{D}^{(1 / n)}\right)^{m} y+b_{m-1}\left({ }^{C} \mathbf{D}^{(1 / n)}\right)^{m-1} y+\cdots+b_{1}{ }^{C} \mathbf{D}^{(1 / n)} y+b_{0} y=0 .
$$


The general solution to (3.12) has the following form:

$$
y=\left.\sum_{k=1}^{l} \sum_{s=0}^{l_{k}} C_{k s}\left(\frac{\mathrm{d}^{s}}{\mathrm{~d} \rho^{s}} E_{\frac{1}{n}, 1}\left(\rho x^{\frac{1}{n}}\right)\right)\right|_{\rho=\rho_{k}},
$$

where $C_{k s} \in \mathbb{C}$ are any constants.

It can be observed that the structure of solution (3.13) to (3.12) mirrors that of ordinary linear differential equations with constant coefficients, where exponential functions are replaced with Mittag-Leffler functions.

\subsubsection{Expression of solution via exponential functions}

Corollary 1. Let us consider the homogeneous linear fractional differential equation with constant coefficients (3.12). Suppose that the roots $\rho_{1}, \ldots, \rho_{m}$ of the characteristic polynomial (3.10) are distinct. Then, the solution (3.13) can be written in the following form:

$$
y=\sum_{s=0}^{n-1} \sum_{k=1}^{m} C_{k} \rho_{k}^{s}\left({ }^{C} \mathbf{I}^{(1 / n)}\right)^{s} \exp \left(\rho_{k}^{n} x\right) .
$$

Proof. Note that the exponential function $\exp (\lambda x), \lambda \in \mathbb{C}$ can be written using basis functions $w_{j n}^{(n)}, j=0,1, \ldots$ :

$$
\exp (\lambda x)=\sum_{j=0}^{+\infty} \lambda^{j} w_{j n}^{(n)} .
$$

It can be observed that selecting $\lambda=\rho^{n}$ yields:

$$
\rho^{s}\left({ }^{C} \mathbf{I}^{(1 / n)}\right)^{s} \exp \left(\rho^{n} x\right)=\sum_{j=0}^{+\infty} \rho^{j n+s} w_{j n+s}^{(n)} .
$$

Thus, (3.2) yields that:

$$
E_{\frac{1}{n}, 1}\left(\rho x^{\frac{1}{n}}\right)=\sum_{s=0}^{n-1} \rho^{s}\left({ }^{C} \mathbf{I}^{(1 / n)}\right)^{s} \exp \left(\rho^{n} x\right) .
$$

If roots of the characteristic polynomial (3.10) are distinct, inserting (3.15) into (3.13) yields (3.14), which finishes the proof.

\subsubsection{Equivalence between fractional differential equations and systems of ODEs}

It can be demonstrated that fractional differential equations can be reduced to systems of ordinary differential equations. Let us consider a special case of (3.12) where $m=n$ :

$$
\left({ }^{C} \mathbf{D}^{(1 / n)}\right)^{n} y+b_{n-1}\left({ }^{C} \mathbf{D}^{(1 / n)}\right)^{n-1} y+\cdots+b_{1}{ }^{C} \mathbf{D}^{(1 / n)} y+b_{0} y=0 .
$$


Note that the solution $y \in{ }^{C} \mathbb{F}_{n}$ can be written in the following form:

$$
y=\sum_{l=0}^{n-1}\left({ }^{C} \mathbf{I}^{(1 / n)}\right)^{l} f_{l},
$$

where $f_{l} \in{ }^{C} \mathbb{F}_{1}$ are series with integer powers of $x$. Inserting (3.17) into (3.16) yields:

$$
\begin{aligned}
& \sum_{j=1}^{n-1} \sum_{l=j}^{n-1} b_{l-j}\left({ }^{C} \mathbf{I}^{(1 / n)}\right)^{j} f_{l}+\sum_{j=1}^{n-1} \sum_{k=n-j}^{n} b_{k}\left({ }^{C} \mathbf{I}^{(1 / n)}\right)^{j}\left(\frac{\mathrm{d}}{\mathrm{d} x} f_{j+k-n}\right) \\
& \quad+\frac{\mathrm{d}}{\mathrm{d} x} f_{0}+\sum_{j=0}^{n-1} b_{j} f_{j}=0 .
\end{aligned}
$$

Rearranging and simplifying (3.18) yields the following system of ordinary differential equations:

$$
\sum_{j=0}^{n-l-1} b_{j} f_{j+l}+\sum_{j=n-l}^{n} b_{j}\left(\frac{\mathrm{d}}{\mathrm{d} x} f_{j+l-n}\right)=0 ; \quad l=0,1, \ldots, n-1 .
$$

Note that this simple example is not the limit of this technique - using (3.17) any fractional differential equation can be converted into a system of ODEs. The solution of this system is then used to obtain the general solution to (3.16).

\subsubsection{Non-homogeneous case}

Let $f=\sum_{j=0}^{+\infty} f_{j} w_{j}^{(n)} \neq 0$ in (3.6). Expanding on the results of the previous subsection, the following statement can be formulated.

Remark 1. The general solution to (3.6) has the following structure:

$$
y=\bar{y}+y^{*},
$$

where

$$
\bar{y}=\left.\sum_{k=1}^{l} \sum_{s=0}^{l_{k}} C_{k s} \frac{\mathrm{d}^{s}}{\mathrm{~d} \rho^{s}} E_{\frac{1}{n}, 1}\left(\rho x^{\frac{1}{n}}\right)\right|_{\rho=\rho_{k}}
$$

is the solution to the associated homogeneous equation (obtained by setting $f=0$ in $(3.6))$ and

$$
y^{*}=\sum_{j=0}^{+\infty} q_{j} w_{j}^{(n)}, \quad q_{j} \in \mathbb{C}
$$

is a particular solution to the nonhomogeneous linear equation (3.6).

Proof. Let $y=\sum_{j=0}^{+\infty} c_{j} w_{j}^{(n)} \in{ }^{C} \mathbb{F}$ be the unknown solution to (3.19). Inserting $y$ into (3.6) and simplifying results in:

$$
\sum_{j=0}^{+\infty}\left(c_{j+m}+b_{m-1} c_{j+m-1}+\cdots+b_{1} c_{j+1}+b_{0} c_{j}-f_{j}\right) w_{j}^{(n)}=0 .
$$


Thus $y$ is a solution to (3.6) if and only if the following recurrence relation holds true:

$$
c_{j+m}+b_{m-1} c_{j+m-1}+\cdots+b_{1} c_{j+1}+b_{0} c_{j}=f_{j}, \quad j=0,1, \ldots
$$

Relation (3.20) defines a non-homogeneous linear recurrence relation with respect to sequence $c_{j}, j=0,1, \ldots$ It is well-known (see, for example, [13]) that the general solution to (3.20) reads:

$$
c_{j}=\sum_{k=1}^{l} \sum_{s=0}^{l_{k}} \gamma_{k s}\left(\begin{array}{l}
j \\
s
\end{array}\right) \rho_{k}^{j-s}+q_{j}, \quad j=0,1, \ldots
$$

The term $q_{j}$ is a particular solution to the recurrence (3.20) and the remaining terms are the general solution to the associated non-homogeneous recurrence relation (3.9).

Inserting (3.21) into $y$ yields (3.19).

Example 1. Consider the following non-homogeneous linear differential equation with constant coefficients:

$$
\begin{aligned}
& \left({ }^{C} \mathbf{D}^{(1 / n)}\right)^{4} y-\left({ }^{C} \mathbf{D}^{(1 / n)}\right)^{3} y-5\left({ }^{C} \mathbf{D}^{(1 / n)}\right)^{2} y-{ }^{C} \mathbf{D}^{(1 / n)} y-6 y \\
& =a_{0}+a_{1} w_{1}^{(n)}+a_{2} w_{2}^{(n)}
\end{aligned}
$$

where $a_{0}, a_{1}, a_{2} \in \mathbb{C}$ are any constants. By the results of Remark 1 , the coefficients of the solution series obey the following linear recurrence:

$$
c_{j+4}-c_{j+3}-5 c_{j+2}-c_{j+1}-6 c_{j}=a_{j}, \quad j=0,1, \ldots,
$$

where $a_{j}=0$ for $j=3,4, \ldots$. The solution to homogeneous part of recurrence (3.23) can be constructed by considering its characteristic polynomial (which is also the characteristic polynomial for the associated homogeneous equation of $(3.22))$ :

$$
P(\rho)=\rho^{4}-\rho^{3}-5 \rho^{2}-\rho-6=0 .
$$

The roots of (3.24) are $\rho_{1,2}= \pm \mathrm{i}, \rho_{3}=-2, \rho_{4}=3$. By Remark 1 , the solution to the homogeneous part of $(3.22)$ reads:

$$
\bar{y}=C_{1} E_{\frac{1}{n}, 1}\left(\mathrm{i} x^{\frac{1}{n}}\right)+C_{2} E_{\frac{1}{n}, 1}\left(-\mathrm{i} x^{\frac{1}{n}}\right)+C_{3} E_{\frac{1}{n}, 1}\left(-2 x^{\frac{1}{n}}\right)+C_{4} E_{\frac{1}{n}, 1}\left(3 x^{\frac{1}{n}}\right) .
$$

Next, a special solution $y^{*}$ to $(3.22)$ must be constructed. This is equivalent to finding a special solution $q_{j}, j=0,1, \ldots$ to recurrence (3.23). Using the method of undetermined coefficients, an initial guess for $q_{j}$ reads:

$$
q_{j}=\nu_{0} 0^{j}+\nu_{1}\left(\begin{array}{l}
j \\
1
\end{array}\right) 0^{j-1}+\nu_{2}\left(\begin{array}{l}
j \\
2
\end{array}\right) 0^{j-2} ; \quad j=0,1, \ldots,
$$

where $\nu_{0}, \nu_{1}, \nu_{2} \in \mathbb{C}$ are undetermined constants. It is clear that $q_{j}=a_{j}=0$ for $j=3,4, \ldots$. Letting $q_{j}=a_{j}$ for $j=0,1,2$ and using (3.23) results in:

$$
\begin{aligned}
-5 \nu_{2}-\nu_{1}-6 \nu_{0} & =a_{0}, \\
-\nu_{2}-6 \nu_{1} & =a_{1}, \\
-6 \nu_{2} & =a_{2} .
\end{aligned}
$$


Solving (3.27) and inserting into (3.26) yields the special solution to (3.22):

$$
y^{*}=-\frac{a_{0}}{6}+\frac{a_{1}}{36}+\frac{29 a_{2}}{216}+\frac{1}{36}\left(a_{2}-6 a_{1}\right) w_{1}-\frac{a_{2}}{6} w_{2} .
$$

Thus, by Remark 1, the general solution to (3.22) is given by combining (3.25) and (3.28):

$$
\begin{aligned}
y & =C_{1} E_{\frac{1}{n}, 1}\left(\mathrm{i} x^{\frac{1}{n}}\right)+C_{2} E_{\frac{1}{n}, 1}\left(-\mathrm{i} x^{\frac{1}{n}}\right)+C_{3} E_{\frac{1}{n}, 1}\left(-2 x^{\frac{1}{n}}\right)+C_{4} E_{\frac{1}{n}, 1}\left(3 x^{\frac{1}{n}}\right) \\
& -\frac{a_{0}}{6}+\frac{a_{1}}{36}+\frac{29 a_{2}}{216}+\frac{1}{36}\left(a_{2}-6 a_{1}\right) w_{1}^{(n)}-\frac{a_{2}}{6} w_{2}^{(n)} .
\end{aligned}
$$

\subsubsection{Cauchy initial value problems}

Corollary 2. Consider the following initial value problem on (3.6):

$$
\left.\left({ }^{C} \mathbf{D}^{(1 / n)}\right)^{k} y\right|_{x=0}=\sigma_{k}, \quad \sigma_{k} \in \mathbb{C}, \quad k=0,1, \ldots, m-1 .
$$

By Remark 1, the solution to (3.6) depends on $m$ undetermined constants $C_{k s}$. Evaluating the solution (3.19) at $x=0$ and using initial conditions (3.29) yields a system of linear equations that can be used to compute the values of $C_{k s}$. Since $\rho_{k} \neq \rho_{l}, k \neq l$, the linear system is consistent and has a unique solution. Thus, the initial value problem (3.6), (3.29) has a unique solution.

Example 2. Consider the initial value problem on (3.23) with $a_{0}=6, a_{1}=-7$, $a_{2}=-6$ and the following initial conditions:

$$
\left.\left({ }^{C} \mathbf{D}^{(1 / n)}\right)^{k} y\right|_{x=0}=\sigma_{k}, \quad k=0,1,2,3
$$

The general solution to (3.23), as constructed in Example 1, reads:

$$
\begin{aligned}
y= & C_{1} E_{\frac{1}{n}, 1}\left(\mathrm{i} x^{\frac{1}{n}}\right)+C_{2} E_{\frac{1}{n}, 1}\left(-\mathrm{i} x^{\frac{1}{n}}\right)+C_{3} E_{\frac{1}{n}, 1}\left(-2 x^{\frac{1}{n}}\right) \\
& +C_{4} E_{\frac{1}{n}, 1}\left(3 x^{\frac{1}{n}}\right)-2+w_{1}^{(n)}+w_{2}^{(n)} .
\end{aligned}
$$

Note that (3.3) yields that for any $\rho \in \mathbb{C}$ :

$$
\left.\left({ }^{C} \mathbf{D}^{(1 / n)}\right)^{k} E_{\frac{1}{n}, 1}\left(\rho x^{\frac{1}{n}}\right)\right|_{x=0}=\rho^{k}, \quad k=0,1, \ldots
$$

Thus, applying initial conditions (3.30) to the general solution (3.31) yields:

$$
\begin{aligned}
C_{1}+C_{2}+C_{3}+C_{4}-2 & =\sigma_{0}, \\
\mathrm{i} C_{1}-\mathrm{i} C_{2}-2 C_{3}+3 C_{4}+1 & =\sigma_{1}, \\
-C_{1}+C_{2}+4 C_{3}+9 C_{4}+1 & =\sigma_{2}, \\
-\mathrm{i} C_{1}+\mathrm{i} C_{2}-8 C_{3}+27 C_{4} & =\sigma_{3} .
\end{aligned}
$$


Solving system(3.32) yields the following constants:

$$
\begin{aligned}
C_{1} & =\left(-\frac{11}{300}-\frac{17 \mathrm{i}}{50}\right) \sigma_{1}-\left(\frac{31}{300}+\frac{17 \mathrm{i}}{300}\right) \sigma_{2}+\left(\frac{1}{50}+\frac{17 \mathrm{i}}{300}\right) \sigma_{3}+\frac{57}{50}+\frac{119 \mathrm{i}}{300}+\frac{1}{2} \sigma_{0}, \\
C_{2} & =\frac{1+\mathrm{i}}{12} \sigma_{1}-\frac{1-\mathrm{i}}{12} \sigma_{2}-\frac{\mathrm{i}}{12} \sigma_{3}+1-\frac{7 \mathrm{i}}{12}+\frac{1}{2} \sigma_{0}, \\
C_{3} & =\left(-\frac{3}{50}-\frac{3 \mathrm{i}}{25}\right) \sigma_{1}+\frac{7-\mathrm{i}}{50} \sigma_{2}-\frac{2}{25}+\frac{7 \mathrm{i}}{50}-\left(\frac{1}{25}-\frac{\mathrm{i}}{50}\right) \sigma_{3}, \\
C_{4} & =\left(\frac{1}{150}-\frac{\mathrm{i}}{50}\right)\left(\mathrm{i} \sigma_{3}+2 \mathrm{i} \sigma_{2}-3-2 \mathrm{i}+\sigma_{2}+2 \sigma_{1}\right) .
\end{aligned}
$$

\subsection{Viability of the presented approach for linear FDEs with variable coefficients}

The fractional power series approach for construction of closed form analytical solutions to fractional differential equations has been illustrated using linear FDEs with constant coefficients. However, the presented technique is not limited to such equations - it can also be used to construct solutions to linear FDEs with variable coefficients or nonlinear problems. It has already been shown for the special case of fractional derivative order $\frac{1}{2}$ in [26] that an approach based on fractional power series can be used to construct solutions to nonlinear fractional differential equations.

Note that it is not always possible to construct closed form solutions to such equations in terms of Mittag-Leffler or other standard functions, but the computation of fractional power series coefficients can always be performed. As an example, let us consider the following linear fractional differential equation with variable coefficients:

$$
\begin{aligned}
& { }^{C} \mathbf{D}^{(1 / n)} y-\sqrt[n]{x} y=\nu, \quad n \in \mathbb{N}, \quad \nu \in \mathbb{R}, \\
& y(0)=A ; \quad A \in \mathbb{R} .
\end{aligned}
$$

Letting $y=\sum_{j=0}^{+\infty} c_{j} w_{j}^{(n)}$ and using previously described techniques yields:

$$
\begin{aligned}
& { }^{C} \mathbf{D}^{(1 / n)} y=\sum_{j=0}^{+\infty} c_{j+1} w_{j}^{(n)} \\
& \sqrt[n]{x} y=\Gamma\left(\frac{1}{n}+1\right) w_{1}^{(n)} \sum_{j=0}^{+\infty} c_{j} w_{j}^{(n)}=\sum_{j=1}^{+\infty} c_{j-1} \frac{\Gamma(j / n+1)}{\Gamma((j-1) / n+1)} w_{j}^{(n)} .
\end{aligned}
$$

Inserting (3.35) and (3.36) into (3.33) results in:

$$
c_{1}-\nu+\sum_{j=1}^{+\infty}\left(c_{j+1}-c_{j-1} \frac{\Gamma(j / n+1)}{\Gamma((j-1) / n+1)}\right) w_{j}^{(n)}=0 .
$$

The above equation together with initial condition (3.34) yields the following relations for coefficients $c_{j}$ :

$$
c_{0}=A, c_{1}=\nu, \ldots, c_{j+1}=c_{j-1} \frac{\Gamma(j / n+1)}{\Gamma((j-1) / n+1)}, \quad j=1,2, \ldots
$$


The solution to recurrence relation (3.37) reads:

$$
\begin{gathered}
c_{2 j}=\frac{\prod_{k=0}^{j-1} \Gamma\left(\frac{2 j-2 k-1}{n}+1\right)}{\prod_{l=1}^{j} \Gamma\left(\frac{2 j-2 l}{n}+1\right)} A ; \quad j=1,2, \ldots, \\
c_{2 j+1}=\frac{\prod_{k=0}^{j-1} \Gamma\left(\frac{2 j-2 k}{n}+1\right)}{\prod_{l=1}^{j} \Gamma\left(\frac{2 j-2 l+1}{n}+1\right)} \nu ; \quad j=1,2, \ldots
\end{gathered}
$$

It is clear that using coefficients (3.38), (3.39) the solution to (3.33) cannot be expressed in closed form using standard functions. However, the presented approach allowed to construct a series solution that can be used to approximate the solution with arbitrary accuracy.

\section{Computational experiments}

\subsection{Fractional damped harmonic oscillator}

Consider the paradigmatic model of the damped harmonic oscillator:

$$
\begin{aligned}
& \frac{\mathrm{d}^{2} z}{\mathrm{~d} x^{2}}-2 \lambda \frac{\mathrm{d} z}{\mathrm{~d} x}+\left(\lambda^{2}+\mu^{2}\right) z=0, \\
& z(0)=A,\left.\quad \frac{\mathrm{d} z}{\mathrm{~d} x}\right|_{x=0}=B
\end{aligned}
$$

where $\lambda, \mu, A, B \in \mathbb{R}$ and $\lambda \pm \mathrm{i} \mu$ are the eigenvalues of (4.1). The general solution to (4.1) reads:

$$
z=A \exp (\lambda x) \cos (\mu x)-\frac{1}{\mu}(\lambda A-B) \exp (\lambda x) \sin (\mu x) .
$$

Now consider the fractional version of (4.1), where the second derivative is replaced with a fractional Caputo derivative of order $m / n, m>n$ :

$$
\left({ }^{C} \mathbf{D}^{(1 / n)}\right)^{m} y-2 \lambda\left({ }^{C} \mathbf{D}^{(1 / n)}\right)^{n} y+\left(\lambda^{2}+\mu^{2}\right) y=0
$$

where $y \in{ }^{C} \mathbb{F}_{n}$. Note that the first order derivation operator $\frac{\mathrm{d}}{\mathrm{d} x}$ is undefined in the algebra ${ }^{C} \mathcal{F}_{n}$, thus it is replaced by the operator $\left({ }^{C} \mathbf{D}^{(1 / n)}\right)^{n}$. The effects of applying $\frac{\mathrm{d}}{\mathrm{d} x}$ or $\left({ }^{C} \mathbf{D}^{(1 / n)}\right)^{n}$ on $z \in{ }^{C} \mathbb{F}_{1}$ are identical.

- The initial conditions (4.2) also define the initial value problem for the fractional differential equation (4.4). Thus, initial conditions (4.2) are rewritten as:

$$
y(0)=A,\left.\quad\left({ }^{C} \mathbf{D}^{(1 / n)}\right)^{n} y\right|_{x=0}=B,\left.\quad\left({ }^{C} \mathbf{D}^{(1 / n)}\right)^{k} y\right|_{x=0}=0,
$$

where $k=1, \ldots, n-1, n+1, \ldots, m$. 
- The values of parameters $\lambda$ and $\mu$ define the equilibrium points of both (4.1) and (4.4). If $\lambda>0$, there exists an unstable node, while the cases $-1<\lambda<0$ and $\lambda \leq-1$ result in a stable spiral and node respectively.

By Lemma 1, the solution to (4.4) reads:

$$
y=\sum_{k=1}^{m} C_{k} E_{\frac{1}{n}, 1}\left(\rho_{k} x\right),
$$

where $\rho_{k}, k=1, \ldots, m$ are the roots of the characteristic polynomial:

$$
\rho^{m}-2 \lambda \rho^{n}+\lambda^{2}+\mu^{2}=0 .
$$

Note that if $m=2 n$ is selected, constants $C_{k}$ can be chosen in such a way to obtain solution (4.3). In the case $\lambda=-0.1, \mu=1$, if $m$ is smaller than $2 n$, it can be observed that the resulting fractional damped harmonic oscillator exhibits smaller amplitudes and the damping comes into effect more quickly (see Figure $3(\mathrm{a})$ ). The opposite effect can be observed when the oscillator is overdamped (for values of $\lambda=-1, \mu=1$ ): the solutions decay more rapidly for values of $\frac{m}{n}$ closer to 2 (see Figure $3(\mathrm{~b})$ ).

Note that equation (4.4) can be transformed into a system of ODEs using the procedure described in subsection 3.2.3. This is discussed in detail in Appendix A.

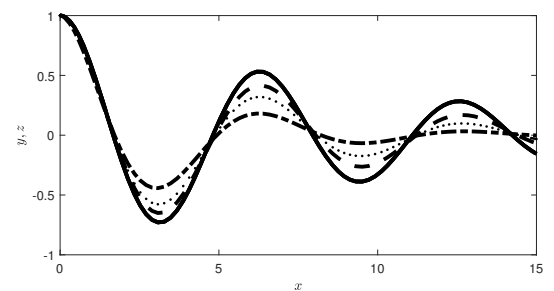

(a)

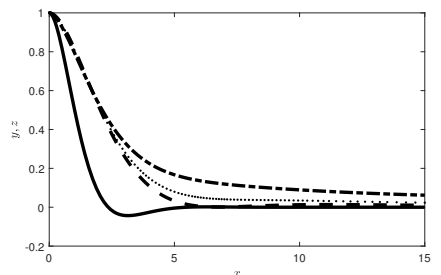

(b)

Figure 3. The solutions to damped harmonic oscillator (solid black line) and fractional damped harmonic oscillator (dashed, dotted and dash-dotted lines represent $n=20,10,5$ respectively) for $\lambda=-0.1, \mu=1, A=1, B=0$ in (a) and $\lambda=-1, \mu=1, A=1, B=0$ in

(b). The derivative order $m$ is set to $2 n-1$ in all cases. It can be seen that as $\frac{m}{n}$ becomes closer to 2 , the solution of the fractional damped harmonic oscillator tends to the non-fractional solution (4.3). Also, for values of $\frac{m}{n}$ significantly smaller than 2 , the damping effect is more powerful in the case $\lambda=-0.1$ (see (a)). However, if $\lambda=-1$, the opposite is true - the solutions decay more slowly when $\frac{m}{n}$ differs most from 2 (see (b)).

\section{Conclusions}

An operator-based approach for the construction of closed-form solutions to fractional differential equations is presented in this paper. The considered technique is a generalization of the results presented in [26] for rational-valued 
fractional derivative order. Caputo and Riemann-Liouville fractional differentiation and integration operators are defined for respective sets of fractional power series.

In order to demonstrate the viability of the proposed technique, explicit expressions of solutions to linear fractional differential equations are obtained in terms of Mittag-Leffler or fractionally-integrated exponential functions. It is also shown that the components of solutions to linear fractional differential equations satisfy associated systems of linear ordinary differential equations.

Even though the operator-based approach is only illustrated using linear fractional differential equations, its applicability to nonlinear problems has already been considered for fractional derivatives of order $\alpha=\frac{1}{2}$ in [26]. The further development of this technique for nonlinear differential equations with rational-valued derivative order is a definite objective of future research.

\section{Acknowledgements}

This research was funded by a grant (No. MIP078/2015) from the Research Council of Lithuania.

\section{References}

[1] O.P. Agrawal. Analytical schemes for a new class of fractional differential equations. J. Phys. A., 40(21):5469-5477, 2007. https://doi.org/10.1088/1751$8113 / 40 / 21 / 001$.

[2] M. Al-Refai, M. Ali Hajji and M.I. Syam. An efficient series solution for fractional differential equations. Abstr. Appl. Anal., ID891837, 2014. https://doi.org/10.1155/2014/891837.

[3] M.K. Al-Srihin and M. Al-Refai. An efficient series solution for nonlinear multiterm fractional differential equations. Discrete Dyn. Nat. Soc., ID5234151, 2017. https://doi.org/10.1155/2017/5234151.

[4] O.A. Arqub, A. El-Ajou and S. Momani. Constructing and predicting solitary pattern solutions for nonlinear time-fractional dispersive partial differential equations. J. Comp. Phys., 293:385-399, 2015. https://doi.org/10.1016/j.jcp.2014.09.034.

[5] O.A. Arqub, A. El-Ajou, Z. Al Zhour and S. Momani. Multiple solutions of nonlinear boundary value problems of fractional order: a new analytic iterative technique. Entropy, 16(1):471-493, 2014. https://doi.org/10.3390/e16010471.

[6] D. Baleanu and J.J. Trujillo. Exact solutions of a class of fractional Hamiltonian equations involving Caputo derivatives. Phys Scr, 80(5):055101, 2009. https://doi.org/10.1088/0031-8949/80/05/055101.

[7] E. Bazhlekova and I. Dimovski. Exact solution of two-term time-fractional Thornley's problem by operational method. Integr. Transf. Spec. F., 25(1):6174, 2014. https://doi.org/10.1080/10652469.2013.815184.

[8] S. Bouzidi, H. Bechir and F. Brémand. Phenomenological isotropic viscohyperelasticity: a differential model based on fractional derivatives. J. Engrg. Math., 99(1):1-28, 2016. https://doi.org/10.1007/s10665-015-9818-6. 
[9] S. Das. Functional Fractional Calculus. Springer, Berlin-Heidelberg, 2011. https://doi.org/10.1007/978-3-642-20545-3.

[10] M. Edelman. Fractional standard map: Riemann-Liouville vs. Caputo. Commun. Nonlin. Sci. Numer. Simulat., 16(12):4573-4580, 2011. https://doi.org/10.1016/j.cnsns.2011.02.007.

[11] A. El-Ajou, O. A. Arqub, Z. Al Zhour and S. Momani. New results on fractional power series: Theories and applications. Entropy, 15(12):5305-5323, 2013. https://doi.org/10.3390/e15125305.

[12] A. El-Ajou, O.A. Arqub, S. Momani, D. Baleanu and A. Alsaedi. A novel expansion iterative method for solving linear partial differential equations of fractional order. Appl. Math. Comp., 257:119-133, 2015. https://doi.org/10.1016/j.amc.2014.12.121.

[13] G. Everest, A. van der Poorten, I. Shparlinski and T. Ward. Recurrence sequences. American Mathematical Society, Providence, RI, 2003. https://doi.org/10.1090/surv/104.

[14] H. Fallahgoul, S. Focardi and F. Fabozzi. Fractional Calculus and Fractional Processes with Applications to Financial Economics: Theory and Application. Academic Press, 2016.

[15] E.F. Doungmo Goufo and J.J. Nieto. Attractors for fractional differential problems of transition to turbulent flows. J. Comput. Appl. Math., 2017.

[16] G.H. Hardy(Ed.). Divergent Series. Clarendon Press, Oxford, 1949.

[17] R. Hilfer. Applications of Fractional Calculus in Physics. World Scientific, Singapore, 2000. https://doi.org/10.1142/3779.

[18] R. Kopka. Estimation of supercapacitor energy storage based on fractional differential equations. Nanoscale Res. Lett., 12(1):636, 2017. https://doi.org/10.1186/s11671-017-2396-y.

[19] S.-D. Lin, C.-H. Lu and S.-M. Su. Particular solutions of a certain class of associated Cauchy-Euler fractional partial differential equations via fractional calculus. Bound. Value Probl., 2013(1):126, 2013. https://doi.org/10.1186/16872770-2013-126.

[20] B.N. Lundstrom, M.H. Higgs, W.J. Spain and A.L. Fairhall. Fractional differentiation by neocortical pyramidal neurons. Nat. Neurosci., 11(11):1335-1342, 2008. https://doi.org/10.1038/nn.2212.

[21] R.L. Magin. Fractional Calculus in Bioengineering. Begell House Redding, 2006.

[22] K.S. Miller and B. Ross(Eds.). An Introduction to the Fractional Calculus and Fractional Differential Equations. Wiley, New York, 1993.

[23] G.M. Mittag-Leffler. Sur la nouvelle fonction $E_{\alpha}(z)$. C. R. Acad. Sci., 137:554558, 1903.

[24] C.A. Monje, Y.-Q. Chen, B.M. Vinagre, D. Xue and V. Feliu. Fractional-order Systems and Controls. Springer, London, 2010. https://doi.org/10.1007/978-184996-335-0.

[25] Z. Navickas and L. Bikulciene. Expressions of solutions of ordinary differential equations by standard functions. Math. Model. Anal., 11:399-412, 2006.

[26] Z. Navickas, T. Telksnys, R. Marcinkevicius and M. Ragulskis. Operatorbased approach for the construction of analytical soliton solutions to nonlinear fractional-order differential equations. Chaos Solitons and Fractals, 104:625-634, 2017. https://doi.org/10.1016/j.chaos.2017.09.026. 
[27] K.B. Oldham and J. Spanier(Eds.). FThe Fractional Calculus: Theory and Applications of Differentiation and Integration to Arbitrary Order. Academic Press, Cambridge, 1974.

[28] F.W.J. Olver, D.M. Lozier, R.F. Boisvert and C.W. Clark. NIST Handbook of Mathematical Functions. Cambridge University Press, Cambridge, 2010.

[29] S.D. Purohit and S.L. Kalla. On fractional partial differential equations related to quantum mechanics. J. Phys. A, 44(4):045202, 2010. https://doi.org/10.1088/1751-8113/44/4/045202.

[30] M. Rivero, L. Rodriguez-Germa and J.J. Trujillo. Linear fractional differential equations with variable coefficients. Appl. Math. Lett., 21(5):892-897, 2008. https://doi.org/10.1016/j.aml.2007.09.010.

[31] T. Sandev, R. Metzler and Ž. Tomovski. Fractional diffusion equation with a generalized Riemann-Liouville time fractional derivative. J. Phys. A, 44(25):255203, 2011. https://doi.org/10.1088/1751-8113/44/25/255203.

[32] S. Tang and Y. Ying. Homogenizing atomic dynamics by fractional differential equations. J. Comput. Phys., 346:539-551, 2017. https://doi.org/10.1016/j.jcp.2017.06.038.

[33] A.M. Tawfik, H. Fichtner, R. Schlickeiser and A. Elhanbaly. Analytical study of fractional equations describing anomalous diffusion of energetic particles. Journal of Physics: Conference Series, IOP Publishing, 869:012050, 2017. https://doi.org/10.1016/j.jcp.2017.06.038.

[34] V.V. Uchaikin and R.T. Sibatov. On fractional differential models for cosmic ray diffusion. Gravitation Cosmol., 18(2):122-126, 2012. https://doi.org/10.1134/S0202289312020132.

[35] B.J. West. Exact solution to fractional logistic equation. Physica A, 429:103108, 2015. https://doi.org/10.1016/j.physa.2015.02.073.

[36] Y. Yu, P. Perdikaris and G.E. Karniadakis. Fractional modeling of viscoelasticity in 3D cerebral arteries and aneurysms. J. Comput. Phys., 323:219-242, 2016. https://doi.org/10.1016/j.jcp.2016.06.038.

\section{Appendix A. Transformation of the fractional damped harmonic oscillator equation into a system of ODEs}

As shown in subsection 3.2.3, the fractional damped harmonic oscillator equation can be transformed into a system of ODEs. Letting $m=2 n-1$ yields the following equation and initial conditions:

$$
\begin{aligned}
& \left({ }^{C} \mathbf{D}^{(1 / n)}\right)^{2 n-1} y-2 \lambda\left({ }^{C} \mathbf{D}^{(1 / n)}\right)^{n} y+\left(\lambda^{2}+\mu^{2}\right) y=0, \\
& y(0)=A ;\left.\quad\left({ }^{C} \mathbf{D}^{(1 / n)}\right)^{n} y\right|_{x=0}=B,\left.\quad\left({ }^{C} \mathbf{D}^{(1 / n)}\right)^{k} y\right|_{x=0}=0,
\end{aligned}
$$

where $k=1, \ldots, n-1, n+1, \ldots, 2 n-2$. The solution to $(0.1),(0.2)$ can be written in the following form:

$$
y=\sum_{k=0}^{n-1}\left({ }^{C} \mathbf{I}^{(1 / n)}\right)^{k} f_{k} ; \quad f_{k}=\sum_{j=0}^{+\infty} a_{j}^{(k)} \frac{x^{j}}{j !} \in{ }^{C} \mathbb{F}_{1} .
$$


Inserting (0.3) into (0.1) results in:

$$
\begin{aligned}
& \sum_{k=0}^{n-1}\left({ }^{C} \mathbf{D}^{(1 / n)}\right)^{2 n-k-2} f_{k}-2 \lambda \sum_{k=0}^{n-1}\left({ }^{C} \mathbf{D}^{(1 / n)}\right)^{n-k} f_{k} \\
& +\left(\lambda^{2}+\mu^{2}\right) \sum_{k=0}^{n-1}\left({ }^{C} \mathbf{I}^{(1 / n)}\right)^{k} f_{k}=0 .
\end{aligned}
$$

Note that

$$
\left({ }^{C} \mathbf{D}^{(1 / n)}\right)^{l}\left({ }^{C} \mathbf{I}^{(1 / n)}\right)^{s} f_{k}=\left({ }^{C} \mathbf{D}^{(1 / n)}\right)^{l-s} f_{k} ; \quad l \geq s
$$

and

$$
\left({ }^{C} \mathbf{D}^{(1 / n)}\right)^{l n} f_{k}=\frac{\mathrm{d}^{l} f_{k}}{\mathrm{~d} x^{l}} ; \quad l=0,1, \ldots
$$

Applying the operator $\left({ }^{C} \mathbf{D}^{(1 / n)}\right)^{n-1}$ to $(0.4)$ and using $(0.5),(0.6)$ on $(0.3)$ yields:

$$
\begin{aligned}
& \sum_{k=1}^{n-1}\left({ }^{C} \mathbf{D}^{(1 / n)}\right)^{n-k-1}\left(\frac{\mathrm{d}^{2} f_{k-1}}{\mathrm{~d} x^{2}}-2 \lambda \frac{\mathrm{d} f_{k}}{\mathrm{~d} x}+\left(\lambda^{2}+\mu^{2}\right) f_{k}\right) \\
& +\left({ }^{C} \mathbf{D}^{(1 / n)}\right)^{n-1}\left(\frac{\mathrm{d} f_{n-1}}{\mathrm{~d} x}-2 \lambda \frac{\mathrm{d} f_{0}}{\mathrm{~d} x}+\left(\lambda^{2}+\mu^{2}\right) f_{0}\right)=0 .
\end{aligned}
$$

Thus, the components $f_{0}, \ldots, f_{n-1}$ of solution (0.3) satisfy the following system of ODEs:

$$
\begin{aligned}
& \frac{\mathrm{d}^{2} f_{k-1}}{\mathrm{~d} x^{2}}-2 \lambda \frac{\mathrm{d} f_{k}}{\mathrm{~d} x}+\left(\lambda^{2}+\mu^{2}\right) f_{k}=0 ; \quad k=1, \ldots, n-1 \\
& \frac{\mathrm{d} f_{n-1}}{\mathrm{~d} x}-2 \lambda \frac{\mathrm{d} f_{0}}{\mathrm{~d} x}+\left(\lambda^{2}+\mu^{2}\right) f_{0} .
\end{aligned}
$$

The initial conditions (0.2) are transformed as follows:

$$
\begin{aligned}
& f_{0}(0)=A ; \quad f_{k}(0)=0 ; \quad k=1, \ldots, n-1, \\
& \left.\frac{\mathrm{d} f_{0}}{\mathrm{~d} x}\right|_{x=0}=B,\left.\quad \frac{\mathrm{d} f_{k}}{\mathrm{~d} x}\right|_{x=0}=0, \quad k=1, \ldots, n-1 .
\end{aligned}
$$

The system $(0.7),(0.8)$ can be solved to obtain the solution to (0.1). 